
CREST Working Paper

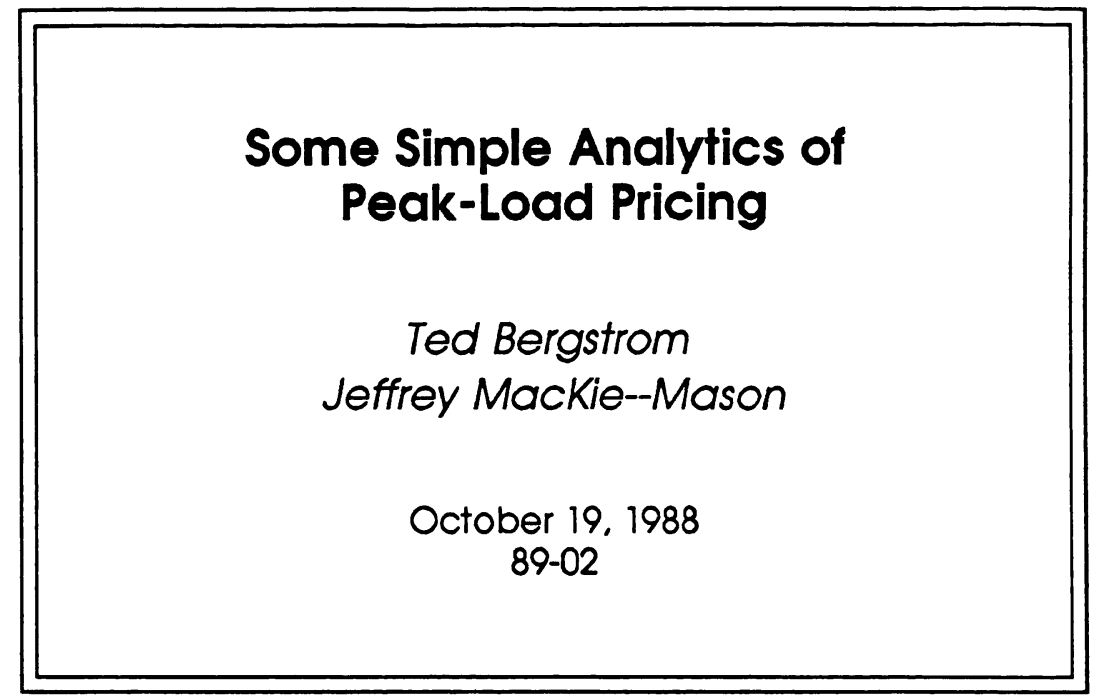

DEPARTMENT OF ECONOMICS University of Michigan Ann Arbor, Michigan 48109
FEB 31989

The Sumner and Laura Foster Librery The Univergity of Migichigan 



\title{
Some Simple Analytics of Peak-Load Pricing
}

\author{
by \\ Ted Bergstrom \\ Jeffrey K. MacKie-Mason \\ University of Michigan \\ University of Michigan and NBER \\ Current version: October 19,1988
}

\begin{abstract}
A bstract. We consider a public utility that provides its service at two different times. Capacity in place can be used in both periods. We study the effects of a change from uniform pricing throughout the day to peak-load pricing, when the utility is constrained to operate with a fixed rate of return on capital. The conventional wisdom seems to be that with peak-load pricing, the peak price will be higher and the off-peak price lower than under uniform pricing, and that peak-load pricing leads to a lower installed capacity. These effects are not generally true. There are plausible cases in which introducing peak-load pricing reduces the price of the service both in peak and off-peak times. Furthermore, peak-load pricing can lead either to greater or to smaller capacity than uniform pricing. We are able to find simple expressions that determine the size and direction of each of these effects. We also provide a criterion for determining whether a particular individual gains or loses from peak-load pricing.
\end{abstract}





\section{Some Simple Analytics of Peak-Load Pricing \\ Ted Bergstrom \\ Jeffrey K. MacKie-Mason}

Consider a public utility that provides its product at two different times, morning and afternoon. Capacity in place can be used both in the morning and in the afternoon, but the amount consumed in either period can be no larger than capacity. Let the afternoon be the "peak" demand period and the morning the "off-peak" period. By this we mean that if the same price is charged for the use of the facility in the morning as in the afternoon, consumers will demand more in the afternoon than in the morning.

We study the effects of a change from uniform pricing throughout the day to peak-load pricing, where the price charged in the morning is lower than that charged in the afternoon. The supplier will be a public utility which is constrained to operate with a fixed rate of return on capital, either by regulation or by competitive forces. The conventional wisdom seems to be that with peak-load pricing, the peak price will be higher and the off-peak price lower than under uniform pricing, and that peak-load pricing leads to a lower installed capacity. This is not generally true. There are plausible cases in which introducing peak-load pricing reduces the price of the service both in peak and off-peak times. Furthermore, peak-load pricing can lead either to greater or to smaller capacity than uniform pricing. We are able to find simple expressions that determine the size and direction of each of these effects.

If preferences differ from person to person, it may be that introducing peak-load pricing is good for some people and bad for others. We explore conditions under which there are both winners and losers and provide a criterion for determining whether an individual has positive or negative willingness to pay for having peak-load pricing.

We do not directly concern ourselves with describing efficient pricing mechanisms. The situation we have in mind is one where costs of metering or conventional practice have led utilities and or regulators to enforce uniform pricing throughout the day. We then ask the question, "ignoring the costs of metering, what will be the allocational and distributional effects of adjusting prices in the direction of peak load pricing?" We were led to this analysis because we were interested in the welfare effects of peak-load pricing schemes in which consumers had the option of choosing whether to install meters that allowed peak-load pricing. ${ }^{1}$ To our surprise, we found that the literature on peak-load pricing lacked an analytical treatment of the basic issues that we treat here. This we think is a necessary starting point for a full analysis of the benefits and costs of metering.

\section{The Basic Model}

Bergstrom's research was supported by a grant from the National Science Foundation. MacKie-Mason gratefully acknowledges financial support from the MIT Center for Energy Policy Research.

1 See MacKie-Mason [1987]. 


\section{Technology}

We begin with a stripped-down model of the technology. Later we will add complexity to this description. We focus on the long-run cost structure, since here we are interested in long run analysis. A public utility company produces its product at two times of day, morning and afternoon. The maximal amount produced in either period can not exceed the "capacity" of the firm, which we denote by $K$. Assume that the size of capacity can be varied at constant unit cost. In addition to capacity costs, the utility faces "user costs" of $u_{A}$ per unit of output in the afternoon, and $u_{M}$ per unit of output in the morning.

\section{Demand}

Let $p_{M}$ and $p_{A}$ denote the prices charged in the morning and the afternoon and let $x_{M}$ and $x_{A}$ denote total consumption in the morning and in the afternoon. It is convenient to assume that preferences are weakly separable between utility services and other goods, and that their preferences over utility services are homothetic. Specifically, every consumer, $i$, is assumed to have preferences representable by a utility function of the form,

$$
U_{i}\left(y^{i}, f\left(x_{M}^{i}, x_{A}^{i}\right)\right)
$$

where $y^{i}$ is $i$ 's consumption of "other goods". Although the functions $U_{i}$ may be different for different consumers, we assume, for now, that the aggregator functions $f\left(x_{M}^{i}, x_{A}^{i}\right)$ are the same for all consumers and that they are homothetic and strictly quasi-concave. Later we consider the case where these aggregators differ.

Let us define $\rho=p_{A} / p_{M I}$. At an interior maximum, an optimizing consumer will choose a consumption bundle such that her marginal rate of substitution between afternoon and morning consumption equals the price ratio, $\rho$. This implies that for all $i$,

$$
\frac{f_{2}\left(x_{M}^{i}, x_{A}^{i}\right)}{f_{1}\left(x_{M}^{i}, x_{A}^{i}\right)}=\frac{p_{A}}{p_{M}}=\rho
$$

Since the functions $f$ are homothetic and strictly quasi-concave, equation (1) uniquely determines the ratio $x_{A}^{i} / x_{M}^{i}$ as a function of $\rho$ for each $i$. Since the functions $f$ are identical, this ratio is the same for everyone. Define the ratio of $x_{A} / x_{M}$ that satisfies equation (1) to be $\chi(\rho)$. The elasticity of substitution between afternoon and morning consumption is defined to be $\sigma(\rho)=-d \ln \chi(\rho) / d \ln \rho$.

We define the expenditure shares

$$
\theta_{A}=\frac{p_{A} x_{A}}{p_{M^{\prime}} x_{M}+p_{A} x_{A}} \text { and } \theta_{M}=\frac{p_{M} x_{M}}{p_{M^{x}}{ }+p_{A} x_{A}} .
$$

Therefore we have $\theta_{A} / \theta_{M}=\rho \chi(\rho)$. Finally, we assume that at uniform prices, demand is greater in the afternoon than in the morning. This can be expressed simply by $\chi(1)>1$. 


\section{Market Structure and Equilibrium}

We assume that the public utility is constrained to operate at a fixed rate of return on capital. This constraint might be enforced by a regulatory agent, or it might be an equilibrium rate of return that is enforced by competition. The capital base on which the utility is allowed to earn this rate of return is proportional to its capacity. Let $c_{K}$ denote the return per unit of capacity that will yield the allowable rate of return on capital. The constraint on the rate of return can be expressed as:

$$
p_{A^{x}}{ }+p_{M} x_{M}=c_{K} K+u_{A} x_{A}+u_{M} x_{M}
$$

We assume that the utility uses its full capacity at least some time during the day, so that when $x_{A}>x_{M}$, it must be that $x_{A}=K$. From this assumption and Equation (2), we deduce that in equilibrium:

$$
p_{A}+\left(p_{M}-u_{M}\right) \frac{x_{M}}{x_{A}}=c_{K}+u_{A}
$$

We further assume that in equilibrium, the utility supplies the quantities $x_{A}$ and $x_{M}$ that meet demand. Then in equilibrium, the two Equations (1) and (3) must be satisfied. In general there will be a one dimensional continuum of solutions to these two equations. But if either the ratio $\rho=p_{A} / p_{M}$ or the ratio $\chi(\rho)=x_{A} / x_{M}$ is specified, then these equations determine a unique solution for each of the variables $p_{A}, p_{M}, x_{A}$, and $x_{M}$. This means, for example, that we can solve for each of these variables under uniform pricing $(\rho=1)$ or under peak-load pricing that equalizes demand in both periods $(\chi(\rho)=1)$. By differentiating the solutions with respect to $\rho$, we can explore the qualitative comparative statics of moving from uniform pricing toward peak-load pricing.

\section{Does peak-load pricing make peak prices rise or fall?}

It is no surprise that changing from uniform pricing to peak-load pricing results in lower prices in off-peak periods. It is less obvious that the price in the peak-load period may also fall. But remember that in equilibrium the rate of return on capacity is fixed. Therefore it might be that if capacity is allocated more efficiently between morning and afternoon use, the equilibrium prices in both periods will fall. To see how this works, consider the special case of zero user costs. If $u_{A}=u_{M}=0$, then from rearranging equation (3), we find that $p_{A}=\left(1-\theta_{M}\right) c_{K}$ where $\theta_{M}$ is the share of the consumer's utility budget that she spends on morning consumption. If the price of off-peak use is lowered, then when substitution is easy $(\sigma>1)$ the shift toward off-peak quantity will be proportionately more than the change in the price ratio. Thus, the share of off-peak sales revenue will increase. But if $\theta_{M}$ increases, it must be that $p_{A}$ will fall.

\section{Two Very Easy Special Cases}

To aid our intuition, we found it helpful to think about two special cases that are easy enough to "solve in one's head". Suppose that user costs are zero and the allowable rate of return on capacity is $c_{K}$. 
Consider the extreme case of easy substitution between morning and afternoon consumption where utility is linear in both periods' consumption but afternoon consumption is "twice as good as morning consumption". That is, suppose that the aggregator function for utility services is:

$$
f\left(x_{A}, x_{M}\right)=x_{A}+2 x_{M} .
$$

If there is a uniform price $p$ in both periods, then the only demand for utility services will be in the afternoon. The zero-profit constraint requires that the entire cost of capacity be repaid by afternoon usage, so that $p=c_{K}$. On the other hand, peak-load pricing in this case must equalize morning and afternoon demands. This will happen when $p_{A}=2 p_{M}$. At these prices, consumers are indifferent between using the service in the morning and afternoon, and consumption in both periods can be set equal to capacity. In effect, peak-load pricing allows the firm to sell its entire capacity twice, once in the morning and once in the afternoon. The profit constraint is satisfied when $p_{A}+p_{M}=c_{K}$. Since $p_{A}=2 p_{M}$, it must be that that with peak-load pricing, $p_{A}=2 c_{K} / 3$, and $p_{M}=c_{K} / 3$. In this example, moving from uniform pricing to peak-load pricing must result, in equilibrium, in lower prices in both periods.

Now consider the opposite extreme-a case of perfect complements, where at any price consumers always want to consume exactly twice as much in the afternoon as in the morning. Let

$$
f\left(x_{A}, x_{M}\right)=\min \left\{x_{A}, 2 x_{M}\right\} .
$$

At any price, consumers will choose $x_{A} / x_{M}=2$. No matter what prices it chooses, the utility can sell all of its capacity in the afternoon and only half of its capacity in the morning. Therefore the zero profit condition will be satisfied for any pair of prices, $p_{A}$ and $p_{M}$, for which $p_{A}+p_{M} / 2=c_{K}$ would satisfy the profit constraint. In this example, raising the price of peak use relative to the price of off-peak use must, in equilibrium, mean an increase in the price of peak use.

\section{A General Answer}

The constraint on rate of return makes it useful to keep track of the contribution of morning sales towards covering capacity costs. Let us define the ratio of net return on morning sales to price of morning consumption as ${ }^{2}$

$$
L_{M}=\left(p_{M}-u_{M}\right) / p_{M}=1-u_{M} / p_{M}
$$

In the appendix, we work out explicit comparative statics effects of moving from uniform pricing toward peak-load pricing. A general statement of our first result is the following.

Theorem 1. Assume technology is as described above and that profits are constrained as in equation (2). Let preferences be weakly separable between utility services and other goods

2 In much of our informal discussion below below, we implicitly assume that $L_{M} \geq 0$. But our equations apply whether $L_{M}$ is positive or negative. Thus it is pnssible to consider cases where regulators require the utility to set the moming price below variable user cost. Wenders (1976) shows that a profit-maximizing utility with a regulated rate of return on installed capital may set the off-peak price beloew marginal cost in order to encourage the expansion of capital intensive base-load capacity. 
and let the aggregator functions for utility services be linearly homogeneous and the same for all consumers. Then for all $\rho$ such that $\chi(\rho)>1$,

$$
\frac{d \ln p_{A}}{d \ln \rho}=\theta_{M}\left(1-L_{M} \sigma(\rho)\right)
$$

and

$$
\frac{d \ln p_{M}}{d \ln \rho}=\frac{d \ln p_{A}}{d \ln \rho}-1=-\left(\theta_{A}+L_{M} \sigma(\rho) \theta_{M}\right) .
$$

Since $f$ is a concave function the elasticity of substitution, $\sigma$, is always positive. From equation (4), it is apparent that the $d p_{A} / d \rho$ is positive or negative depending on whether $1-L_{M} \sigma$ is positive or negative. From equation (5), we see that $d p_{M} / d \rho$ is always negative. Therefore we can assert:

Corollary 1. Under the assumptions of Theorem 1, moring toward peak-load pricing results in: (a) lower prices in both peak and off-peak times if the elasticity of substitution between peak and off-peak consumption is greater than $p_{M I} /\left(p_{M}-u_{M}\right)$. (b) higher prices in peak times and lower prices in off-peak times if the elasticity of substitution is less than $p_{M} /\left(p_{M}-u_{M}\right)$.

3. Does peak-load pricing increase or decrease industry capacity?

The conventional wisdom seems to be that peak-load pricing will reduce the demand for capital by utilities (see, e.g., Berlin et al. (1974)). We shall demonstrate, to the contrary, that under quite plausible circumstances-iiz., a long-run demand elasticity greater than unity - that the equilibrium capacity may increase under peak-load pricing.

There are two forces at work here. Peak-load pricing allow's more efficient use of capacity, since less capacity is idle off-peak. This means that less capacity is required to generate a given amount of the composite commodity, utility services, appropriately defined. On the other hand utility services become cheaper, which tends to increase the demand for utility services. It turns out that which effect exerts the stronger force on equilibrium capacity depends simply on the elasticity of demand for utility services.

The weakly separable functional form that we have assumed for utility allows us to define a composite commodity, $x$, such that the quantity of $x$ is $f\left(x_{M}, x_{A}\right)$ and the "price" $p$ of the composite commodity is just equal to the value of the expenditure function,

$$
p=e\left(p_{M}, p_{A}\right)=\min _{f\left(x_{M}, x_{A}\right)=1} p_{M} x_{M}+p_{A^{\prime}} x
$$

Since the equilibriur conditions determine $p_{A}$ and $p_{M}$ as functions of $\rho$, we can define

$$
p(\rho)=e\left(p_{M}(\rho), p_{A}(\rho)\right) .
$$

Applying standard results from duality theory, we find that

$$
\frac{d \ln p(\rho)}{d \ln \rho}=\theta_{A} \frac{d \ln p_{A}}{d \ln \rho_{5}}+\left(1-\theta_{A}\right) \frac{d \ln p_{M}}{d \ln \rho}
$$


Substituting from equations (4) and (5) into equation (8) we find that:

$$
\frac{d \ln p(\rho)}{d \ln \rho}=-L_{M} \sigma(\rho)\left(1-\theta_{A}\right) .
$$

From equation (9) we are able to conclude the following:

Remark 1. Under the assumptions of Theorem 1, if demand in the afternoon exceeds demand in the morning at the price ratio $\rho=p_{A} / p_{M}$, then moving toward peak-load pricing must lower the price of the composite good.

This result is not surprising, since an increase in $\rho$ tends to equalize demand in the morning and afternoon, thus allowing more "efficient" production of the composite commodity. But whether a reduction in its price results in an increase or a decrease in expenditure on the commodity depends on the magnitude of the elasticity of demand.

Let total demand for the composite commodity, $x$, be $D_{\boldsymbol{x}}(\boldsymbol{p})$ where $p$ is the price of the composite commodity. Denote the price elasticity for the composite commodit $y$ by $\eta$. Total revenue, $p_{A} x_{A}+p_{M^{x}}{ }_{M}$, from the sales of utility services is equal to $p D_{x}(p)$. Therefore when $\chi(\rho)>1$, so that $K=x_{A}$, we can write

$$
\theta_{A}=\frac{p_{A} K}{p(\rho) D_{x}(p(\rho))}
$$

Logarithmically differentiating both sides of (10) and making substitutions, we are able to prove the following:

Theorem 2. Under the assumptions of Theorem 1,

$$
\frac{d \ln K}{d \ln \rho}=-\theta_{M} \sigma\left(1-L_{M I} \eta\right. \text {. }
$$

From Remark 1 and Theorem 2, if follows that:

Corollary 2. Under the assumptions of Theorem 1, moving toward peak-load pricing will lower the price of the composite good, utility services, and will increase or decrease the equjlibrium capacity depending on whether the absolute value of the price elasticity of demand for the composite good is greater or smaller than $p_{M} /\left(p_{M}-u_{M}\right)$.

Again in case $u_{M}=u_{A}=0$, it is pretty easy to interpret this result. Since the composite price $p$ falls when prices move toward peak-load pricing, total consumer expenditures must increase if the aggregate elasticity is greater than one. Since the rate of return on capacity is constrained to stay constant and since user costs are zero it must be that in equilibrium the extra consumer expenditures are spent on more capacity. If user costs are positive, then lowering the price increases off-peak utilization of the capacity which increases the total off-peak user costs. Only when the demand elasticity is large enough relative to the user cost effect $\left(-\eta>1 / L_{M}\right)$ will an increase in total expenditure require a higher equilibrium capacity. 


\section{Who Gains And Who Loses From Peak-Load Pricing?}

In the last section we showed that when preferences over time of use are homothetic and identical, moving from uniform pricing toward time-of-day pricing will reduce the cost of the composite good, "utility services" for every consumer. So then, all consumers benefit from the change. Now suppose that preferences differ between individuals. If the prices of morning and afternoon consumption both fall as the system is moved toward peak-load pricing, then of course all consumers will benefit. But if the price of afternoon consumption rises and the price of morning consumption falls, then those for whom an especially large proportion of consumption is in the afternoon might be worse off.

To analyze these effects, we allow different consumers to have different aggregator funtions, $f_{i}\left(x_{M}, x_{A}\right)$. We assume utility functions are of the form, $U_{i}\left(y^{i}, f^{i}\left(x_{M}, x_{A}\right)\right)$, and that the functions $f^{i}\left(x_{M}, x_{A}\right)$ are homogeneous of degree one. We also assume that the ratio $\chi$ of total afternoon demand to total morning demand is determined by the ratio $\rho$ of the afternoon price to the morning price. ${ }^{3}$ Then, just as in the earlier sections, we can define the elasticity of substitution to be $\sigma(\rho)=-d \ln \chi(\rho) / d \ln \rho$.

The response of the equilibrium prices $p_{M}$ and $p_{A}$ to changes in $\rho$ is still described by equations (4) and (5). This makes it easy to figure out whether a consumer is a net gainer or a net loser from the price change. Since the aggregator functions $f_{i}$ are assumed to be homothetic, all we need to do to find out whether $i$ is a gainer or a loser is to see whether the unit cost to $i$ of producing one unit of the aggregate $f_{i}\left(x_{A}, x_{M}\right)$ has gone up or down. This cost is measured by the expenditure function,

$$
p_{i}(\rho)=e_{i}\left(p_{M}(\rho), p_{A}(\rho)\right)=\min _{f_{i}\left(x_{M} \cdot x_{A}\right)=1}\left\{p_{M I}(\rho) x_{M}+p_{A}(\rho) x_{A}\right\}
$$

From standard duality results.

$$
\frac{d \ln p_{i}(\rho)}{d \ln \rho}=\theta_{A}^{i} \frac{d \ln p_{A}}{d \ln \rho}+\theta_{M}^{i} \frac{d \ln p_{M}}{d \ln \rho} .
$$

where $\theta_{A}^{i}$ and $\theta_{M}^{i}$ are the afternoon's and morning's shares of $i$ 's expenditures on utility services. Substituting from equations (4) and (5) into (12) and rearranging terms, we find that

$$
\frac{d \ln p^{i}(\rho)}{d \ln \rho}=\theta_{M}\left(1-\sigma(\rho) L_{M}\right)-\theta_{M}^{i} .
$$

From equation (13) we can see the following:

Theorem 3. If preferences are as modelled in this section and technology and market structure are as modelled in Section 1, then a movement toward peak-load pricing will benefit consumer $i$ if

$$
\frac{\theta_{M}^{i}}{\theta_{M}}>1-\sigma(\rho) L_{M}
$$
3 If, for example, utility takes the quasi-linear form, $U_{i}\left(y^{i}, f^{i}\left(x_{M}, x_{A}\right)\right)=y^{i}+f^{i}\left(x_{M}, x_{A}\right)$, this assumption will be
satisfied. 
and will make consumer $i$ worse off if the inequality is reversed.

Theorem 3 gives us an exact description of who gains and who loses from peak-load pricing. From Theorem 3, we can also deduce some simple rules of thumb for determining losers and gainers. In particular, we have

Corollary 3. Under the conditions of Theorem 3, a move toward peak-load pricing will:

(a) yield a Pareto improvement if $\sigma L_{M}>1$;

(b) will benefit customer $i$ for any substitution elasticity $\sigma$ if $i$ has a higher than average off-peak expenditure share $\left(\theta_{M}^{i}>\theta_{M}\right)$ and if $L_{M} \geq 0$.

\section{More General Technologies}

\section{Different Types of Plant in Peak and Off-Peak Periods}

If a utility company does not use its full capacity in both the morning and the afternoon, there may be cost advantages in having one type of plant that is used all day and a second type of plant that is used only for the afternoon peak load. Such an arrangement would be particularly appealing if it is possible to reduce user costs by building a more expensive type of plant. Then it may be desirable to employ a high-capacity-cost, low-user-cost plant for all-day use and a cheaper plant with higher user costs for peak-load use. ${ }^{4} 5$

As it happens, there is a very nice isomorphism between models of this type and the model we have already treated. Let us suppose that a utility has access to two kinds of capacity, one of which it uses all day and one of which it uses only in the afternoon. Then the utility uses $K_{M}=x_{M}$ units of the first kind of capacity and $K_{A}=x_{A}-x_{M}$ units of the second type. Let $c_{K}^{M}$ and $c_{K}^{A}$ be the costs per unit of capacity of the two types and let $u_{M}$ and $u_{A}$ be the corresponding user costs. Then total costs to the utility of supplying $\left(x_{M}, x_{A}\right)$ are :

$$
c_{K}^{M} x_{M}+c_{K}^{A}\left(x_{A}-x_{M}\right)+u_{M} x_{M}+u_{A^{x}} x_{A}=\left(c_{K}^{A}+u_{A}\right) x_{A}+\left(c_{K}^{M}-c_{K}^{A}+u_{M}\right) x_{M} .
$$

Compare this expression to total costs in the model where there is only one kind of capacity. In this case, total costs are just

$$
\left(c_{K}^{A}+u_{A}\right) x_{A}+u_{M} x_{M}
$$

Looking at expressions (15) and (16), we see that we could write the costs for (15) in the form of (16) by defining $\tilde{u}_{M}=c_{K}^{M}-c_{K}^{A}+u_{M}$. Indeed all of our analysis of the previous

4 This type of technology has been discussed by (Turvey (1968), Joskow (1976), and Wenders (1976), among others.)

5 A closely related possibility is that as technological change occurs, a utility company finds itself with some old capacity with high user costs and some newer capacity with lower user costs. The company may then choose to use the capacity with high user costs only at peak-load times. We will not explicitly deal with this case here, but will confine our attention to long-run equilibrium analysis. But certainly it would be possible to extend our results in this direction. 
sections goes through for this model. All of our previous results therefore apply to this model if one simply reinterprets the user cost in the earlier model to be $\dot{u}_{M}$, which is the actual user cost plus the difference between the unit cost of capacity which is used all day and the unit cost of capacity which is used only in the afternoon. In retrospect, it is easy to see why this must be. In the earlier model the only marginal cost of selling more utility services in off-peak periods was the user cost. In the current model, the long-run cost of expanding morning output includes both the user cost and the cost of replacing one kind of capacity with the other. ${ }^{6}$

One more remark is in order about this isomorphism. Our results would be formally correct, even if it turned out that $\bar{u}_{M}$ were negative. But if this were the case, we would have to be careful in interpreting the theorems since we accustomed to almost automatically assuming that marginal costs are nonnegative. As it turns out, in reasonable models of longrun equilibrium there is a presumption that $\dot{u}_{M} \geq 0$. In fact, it is reasonable to assume that $c_{M}+u_{M} \leq c_{A}+u_{A}$, since otherwise it would be profitable to use the "all-day technology" even for plants that are used only in the morning. From this inequality it follows that $\bar{u}_{M}=c_{M}-c_{A}+u_{m} \geq u_{A}$ and therefore if $u_{A} \geq 0$, then $\tilde{u}_{M} \geq 0$.

\section{Nonconstant Returns in Provision of Capacity}

It is not too difficult to extend our results to cases where there are increasing or decreasing returns to scale in the provision of capacity. We do not work out this story in detail, but simply sketch its outlines. To enable us to concentrate on the effects of scale without obscuring details, we will confine our attention to the case where there is a single kind of capacity and where user costs are zero. Let us also suppose that the firm is constrained to make zero profits. ${ }^{\top}$

Let us write $c_{K}(K)$ to denote the average cost of capacity when capacity is $K$ and let us define $\epsilon_{K}\left(K^{\prime}\right)$ to be the elasticity of average cost with respect to $K$. That is, ${ }{ }_{K}(K) \equiv$ $d \ln c_{K}\left(K^{\prime}\right) / d \ln K$. Then $\epsilon_{K}(K)$ will be positive if there is increasing average cost and negative if there is decreasing average cost. Assuming that the marginal cost of capacity is always nonnegative, we must have $\epsilon_{K}(K)>-1$ for all $K$.

Taking into account our assumption that user costs are zero and that the average cost of capacity is now $c_{K}(K)$, the equilibrium condition in equation (3) can be rewritten as

$$
p_{A}+\frac{p_{M} x_{M}}{x_{A}}=c_{K}(K)
$$

A simple calculation shows that equation (17) is equivalent to

$$
p_{A}=c_{K}(K) \theta_{A} .
$$

6 There is another simple isomorphism that is worth pointing out. Formally, in either of these models, there is no reason to distinguish between capacity costs in the peak period and user costs in the peak period. Either model would be isomorphic to o model with zero user costs in the afternoon and with afternoon capacity costs $i_{K}^{A}=c_{K}^{A}+u_{M}$. Williamson (1966) has noticed this simplification in the modelling of long-run utility costs.

7 Our results apply as well to the case of a firm regulated to profits which are a fixed proportion of capacity costs. 
We can logarithmically differentiate to obtain:

$$
\frac{d \ln p_{A}}{d \ln \rho}=\frac{d \ln \theta_{A}}{d \ln \rho}+\epsilon_{K}(K) \frac{d \ln K}{d \ln \rho} .
$$

Using results and methods from previous sections, we find that:

Theorem 4. Under the assumptions of this section,

$$
\frac{d \ln K}{d \ln \rho}=-\theta_{M} \sigma(\rho) \frac{1+\eta}{1-\eta \epsilon_{K}(K)} .
$$

It is trivial to show that $1-\eta \epsilon_{K}(K)>0$ is necessary for an equilibrium to exist; if the inequality is reversed, then the economies of scale in capacity and the demand responsiveness of the composite good demand would feed on each other to drive equilibrium output to inifinity. Thus, it is clear that even when costs are non-constant, the result of Corollary 2 continues to hold: the equilibrium capacity will increase or decrease depending on whether the absolute value of the price elasticity is greater or smaller than one. ${ }^{8}$

The effect of nonconstant costs is to magnify or diminish the equilibrium capacity change, as the cost elasticity is negative or positive. For instance, if $\epsilon_{K}<0$ then the denominator of (20) is less than one, and the change in $K$ is magnified. If demand is elastic $(\eta<-1)$, capacity will increase more the larger are the cost economies (negative $\epsilon_{K}$ ). The reason is simple: the price efficiency gain from peak-load pricing which leads better-off consumers to purchase more utility services is amplified by the cost efficiency gain as scale economies are achieved (and prices correspondingly decreased; see below).

We can now substitute equation (20) into (19), to find:

Theorem 5. Under the assumptions of Theorem 4.

$$
\frac{d \ln p_{A}}{d \ln \rho}=\theta_{M}\left(1-\sigma(\rho) \frac{1+\epsilon_{K}(K)}{1-\eta \epsilon_{K}\left(K^{\prime}\right)}\right)
$$

and

$$
\frac{d \ln p_{M}}{d \ln \rho}=-\left(\theta_{A}+\theta_{M} \sigma(\rho) \frac{1+\epsilon_{K}(K)}{1-\eta \epsilon_{K}(K)}\right) .
$$

For completeness, we present without proof the comparative statics results when capacity costs $\left(c_{K}\right)$ are non-constant and off-peak user costs $\left(u_{M}\right)$ are non-zero:

Theorem 6. Under the assumptions of Theorem 1, with the additional assumption that unit capacity costs may be a non-constant function of capacity; $c_{K}(K)$. moving towards peak-load pricing results in

$$
\frac{d \ln p_{A}}{d \ln \rho}=\theta_{M}\left(1-\sigma(\rho) \frac{L_{M}+\hat{\epsilon}_{K}(K)}{1-\eta \hat{\epsilon}_{K}(K)}\right)
$$

8 Note that with $u_{M}=0$, we have $L_{M}=1$. 


$$
\begin{aligned}
& \frac{d \ln p_{M}}{d \ln \rho}=-\left(\theta_{A}+\theta_{M} \sigma(\rho) \frac{L_{M}+\hat{\epsilon}_{K}(K)}{1-\eta \hat{\epsilon}_{K}(K)}\right) \\
& \frac{d \ln K}{d \ln \rho}=-\sigma(\rho) \theta_{M}\left(\frac{1+L_{M} \eta}{1-\eta \hat{\epsilon}_{K}(K)}\right)
\end{aligned}
$$

u'here

$$
\hat{\epsilon}_{K}(K)=\epsilon_{K}(K) \frac{\left(p_{M}-u_{M}\right) x_{M}+p_{A} x_{A}}{p_{M} x_{M}+p_{A} x_{A}}
$$

\section{Discussion}

There is already a large literature on peak-load pricing. However, that literature does not appear to work out the basic equilibrium implications for prices and capacity when peak-load pricing is adopted by a rate-of-return regulated utility.

One branch of the literature is primarily concerned with the optimal technology mix for producing a utility service facing time-varying demand; Joskow (1976) cogently summarizes this literature. Given an efficient mix of base and peak-period technologies, many authors work out variations of the Ramsay rules for optimal multi-commodity pricing subject to a profit constraint (e.g., Steiner (1957); Turvey (1968); Williamson [1966]; Boiteux [1964]; Baumol and Bradford [1970]). We could not find a careful comparison of the Ramsay prices to equal prices, nor a treatment of the effects of Ramsay prices on equilibrium demands and capacities.

Another strand in the literature concerns optimal utility pricing when some information about individual preferences is unavailable to the utility or regulators (e.g.. Willig [1979]; Mirman and Sibley [1980!). Much of this literature ignores time-of-day issues altogether, focusing instead on the design of pricing schedules subject to self-selection constraints.

We have nothing new to add to the standard welfare analysis of this problem. Straightforward application of the Kuhn-Tucker theorem tells us that in the absence of costs for metering, an efficient pricing system would either equalize demand in the morning and afternoon or, if demand in one period is smaller than demand in the other, then in the off-peak period, price is equal to user costs. We are interested in the comparative statics of situations which are not optimal in this sense. This may occur because regulatory agencies are maintaining a system of cross-subsidization in response to political forces or it may be because it has been decided that costs of metering are too high to make peak-load pricing worthwhile. In either case it is important to know the allocational and distributional effects of movements in the direction of peak-load pricing if policy makers are considering such changes.

We have pointed out that, contrary to casual intuition, peak-load pricing may increase rather than decrease the installed capacity base. This result may be the opposite of what some policy makers intend when they propose peak-load pricing. Other effects we identify in our general equilibrium analysis are sometimes ignored in applied welfare analyses, possibly introducing serious biases to the calculations of the net welfare gain from peak-load pricing.

For example, income effects are often dismissed with an argument that when one price 
increases and the other falls, the income effect on average will be small. We have shown that the premise of divergence up and down from the uniform price may not be correct in equilibrium. As another example, $e x$ ante revenue neutrality is sometimes imposed on the welfare analysis with the assertion that it is equivalent to a regulated rate of return on capacity. This equivalence fails to hold if equilibrium installed capacity differs after a move to peak-load prices, or if shadow capacity costs are non-constant.

The seriousness of the errors possible when some of the equilibrium effects are ignored is illustrated by Caves et al. [1984]. In an evaluation of a time-of-use electricity pricing experiment in Wisconsin, they calculate a producer plus consumer welfare increase of $\$ 15$ million. If they intentionally ignore production cost effects the result changes by one-third. Ignoring income effects (using a surplus rather than an exact welfare measure) led to greater errors.

As public utilities and their regulators seek to determine whether or not the particular circumstances they face merit a move towards time-of-use pricing, they will need to know the answers to the questions we have posed: what will happen to the prices in the different periods; what will happen to equilibrium capacity; and who will benefit, who will lose? 


\section{Appendix}

The results in this appendix are all proved under the assumptions made for Theorem 1 of the text, unless otherwise indicated.

Fact 1. Where $\sigma(\rho)$ is the (negative of the) elasticity of substitution between $x_{M}$ and $x_{A}$, at the price ratio, $\rho$,

$$
\frac{d \ln \theta_{A}}{d \ln \rho}=\theta_{M}(1-\sigma(\rho))
$$

Proof. The utility function is of the form $U_{i}\left(y^{i}, f\left(x_{M}, x_{A}\right)\right.$, where $f\left(x_{M}, x_{A}\right)$ is strictly quasi-concave and homogeneous of degree one. Consumer optimization therefore implies that:

$$
\frac{f_{2}\left(x_{M}^{i}, x_{A}^{i}\right)}{f_{1}\left(x_{M}^{i}, x_{A}^{i}\right)}=\rho
$$

where $\rho=p_{\text {. }}: p_{M I}$.

Since $f$ is strictly quasi-concave, the slope of an isoquant is uniquely determined by the ratio $x_{A} / x_{M}$. Therefore the equilibrium condition (A.1) uniquely determines the ratio $x_{A} / x_{M}=\chi(\rho)$. Evidently

$$
\rho \chi(\rho)=p_{A} x_{A} / p_{M} x_{M}=\theta_{A} /\left(1-\theta_{A}\right) .
$$

It follows that $\theta_{A}=\rho \chi(\rho) /(1 \div \rho \chi(\rho))$. Therefore equilibrium values of $\theta_{A}$ are uniquely determined by $\rho$, and we can define the function $\theta_{A}(\rho)$ accordingly.

Most of the results are more conveniently derived in the form of elasticities, so we work largely with logarithmic derivatives. From (A.2), it follows that

$$
\ln \theta_{A}-\ln \left(1-\theta_{A}\right)=\ln \rho+\ln \chi(\rho) \text {. }
$$

The elasticity of substitution is $\sigma(\rho)=-d \ln \chi(\rho) / d \ln (\rho)$. Differentiating both sides of equation (A.3) with respect to $\ln \rho$, we have

$$
\frac{d \ln \theta_{A}}{d \ln \rho}\left(1+\frac{\theta_{A}}{1-\theta_{A}}\right)=1-\sigma(\rho) .
$$

Multiply both sides of (A.4) by $\left(1-\theta_{A}\right)$ and recall that $\left(1-\theta_{A}\right)=\theta_{M}$ to obtain Fact 1 .

Theorem 1. For all, such that $\chi(\rho)>1$,

$$
\frac{d \ln p_{A}}{d \ln \rho}=\theta_{M}\left(1-L_{M} \sigma(\rho)\right)
$$

and

$$
\frac{d \ln p_{M}}{d \ln \rho}=\frac{d \ln p_{A}}{d \ln \rho}-1=-\left(\theta_{A}+L_{M} \sigma(\rho) \theta_{M}\right)
$$


Proof. If we multiply both sides of text equation (2) by $\theta_{A} / x_{A}$, we have:

$$
p_{A}=\left(c_{K}+u_{A}+u_{M} \frac{x_{M}}{x_{A}}\right) \theta_{A} .
$$

From the fact that $\theta_{M}=1-\theta_{A}$, it follows that $\frac{x_{M A} \theta_{A}}{x_{A}}=\rho\left(1-\theta_{A}\right)$. Therefore equation (A.5) is equivalent to

$$
p_{A}=\left(c_{K}+u_{A}-u_{M} \rho\right) \theta_{A}+u_{M} \rho .
$$

Differentiating, we have:

$$
\frac{d p_{A}}{d \rho}=\left(c_{K}+u_{A}-u_{M} \rho\right) \frac{d \theta_{A}}{d \rho}+u_{M}\left(1-\theta_{A}\right) .
$$

Then multiplying both sides by $\rho / p_{A}$ and simplifying, we have:

$$
\frac{d \ln p_{A}}{d \ln \rho}=\left(c_{K}+u_{A}-u_{M} \rho\right) \frac{\theta_{A}}{p_{A}} \frac{d \ln \theta_{A}}{d \ln \rho}+\frac{u_{M}}{p_{A}} \rho\left(1-\theta_{A}\right) .
$$

From (A.6) and the definition of $L_{M}$, we see that

$$
\left(c_{K}+u_{A}-u_{M} \rho\right) \frac{\theta_{A}}{p_{A}}=1-\frac{u_{M}}{p_{A}} \rho=1-\frac{u_{M}}{p_{M}}=L_{M} .
$$

Therefore, using Fact 1 and (A.8) we can simplify (A.7) to

$$
\frac{d \ln p_{A}}{d \ln \rho}=L_{M} \theta_{M}(1-\sigma(\rho))+\frac{u_{M}}{p_{A}} \rho\left(1-\theta_{A}\right) .
$$

Since $\frac{u_{M}}{p_{A}} \rho\left(1-\theta_{A}\right)=\left(1-L_{M}\right) \theta_{M}$, we can further simplify (A.9) to

$$
\frac{d \ln p_{A}}{d \ln \rho}=\theta_{M}\left(1-L_{M} \sigma(\rho)\right)
$$

This is the first equation claimed in Theorem 1 . The second equation is a trivial consequence of the first.

Theorem 2. Under the assumptions of Theorem 1,

$$
\frac{d \ln K}{d \ln \rho}=-\theta_{M} \sigma(\rho)\left(1+L_{M} \eta\right)
$$

Proof. We logarithmically differentiate text equation (10) to get

$$
\begin{aligned}
\frac{d \ln K}{d \ln \rho} & =\frac{d \ln \theta_{A}}{d \ln \rho}-\frac{d \ln p_{A}}{d \ln \rho}+\frac{d \ln p}{d \ln \rho}+\frac{d \ln D_{x}}{d \ln p} \frac{d \ln p}{d \ln \rho} \\
& =\frac{d \ln \theta_{A}}{d \ln \rho}-\frac{d \ln p_{A}}{d \ln \rho_{14}}+\frac{d \ln p}{d \ln \rho}(1+\eta) .
\end{aligned}
$$


Substituting the results of Fact 1 and Theorem 1, and text equation (9), yields the result.

Theorem 4. Under the assumptions of this section,

$$
\frac{d \ln K}{d \ln \rho}=-\theta_{M} \sigma(\rho) \frac{1+\eta}{1-\eta \epsilon_{K}(K)} .
$$

Proof. Since we now assume that user costs are zero but capacity costs are non-constant, we can rewrite (A.5) as

Logarithmically differentiating,

$$
p_{A}=c_{K}(K) \theta_{A}
$$

$$
\begin{aligned}
\frac{d \ln p_{A}}{d \ln \rho} & =\frac{d \ln \theta_{A}}{d \ln \rho}+\frac{d \ln c_{K}(K)}{d \ln K} \frac{d \ln K}{d \ln \rho} \\
& =\theta_{M}(1-\sigma(\rho))+\epsilon_{K}(K) \frac{d \ln K}{d \ln \rho}
\end{aligned}
$$

where the second equality follows from using Fact 1 and the definition of the cost elasticity, $\epsilon_{K}$. Then, since $\rho=p_{.4} / p_{M}$, we know that

so

$$
\frac{d \ln p_{M}}{d \ln \rho}=\frac{d \ln p_{A}}{d \ln \rho}-1
$$

$$
\frac{d \ln p_{M}}{d \ln \rho}=-\theta_{A}-\sigma \theta_{M}+\epsilon_{K}(K) \frac{d \ln K}{d \ln \rho}
$$

by substituting (A.12). Then we can rewrite text equation (8) as

$$
\begin{aligned}
\frac{d \ln p(\rho)}{d \ln \rho} & =\theta_{A}\left[\frac{d \ln p_{A}}{d \ln \rho}-\frac{d \ln p_{M}}{d \ln \rho}\right]+\frac{d \ln p_{M}}{d \ln \rho} \\
& =\theta_{A}+\frac{d \ln p_{M}}{d \ln \rho} \\
& =-\sigma \theta_{M}+\epsilon_{K}(K) \frac{d \ln K}{d \ln \rho}
\end{aligned}
$$

where the second equality follows from (A.13) and the third equality from substituting (A.14).

We now need to find the change in $K$. We can begin with equation (A.10). Substituting in (A.12) gives

$$
\begin{aligned}
\frac{d \ln K}{d \ln \rho} & =-\epsilon_{K}(K) \frac{d \ln K}{d \ln \rho}+(1+\eta) \frac{d \ln p(\rho)}{d \ln \rho} \\
& =-\sigma \theta_{M}(1+\eta)+\eta \epsilon_{K}(K) \frac{d \ln K}{d \ln \rho} \\
& =-\sigma \theta_{M} \frac{1+\eta}{1-\eta \epsilon_{K}(K)}
\end{aligned}
$$

using (A.15) to get the second equality and rearranging to obtain the third. Equation (A.16) completes the proof of Theorem 4.

\section{Proof of Theorem 5.}

The results follow trivially from substituting (A.16) into equations (A.12) and (A.14). 


\section{References}

Baumol, W. J. and D. F. Bradford (1970) "Optimal Departures from Marginal Cost Pricing," American Economic Review, 60, 265-283.

Berlin, E., C. J. Cicchetti, and W. J. Gillen. (1974) Perspectives on Power. Cambridge: Ballinger Publ. Co..

Boiteux (1964) "The Choice of Plant and Equipment for the Production of Electric Energy," in Marginal Cost Pricing in Practice, ed. James Nelson. Englewood Cliffs, N.J.: Prentice Hall.

Caves, D. W., L. R. Christensen, P. E. Schoech, and W. Hendricks (1984) "A Comparison of Different Methodologies in a Case Study of Residential Time-of-Use Electricity Pricing: Cost-Benefit Analysis," Journal of Econometrics, 26, 17-34.

Joskow, P. (1976) "Contributions to the Theory of Marginal Cost Pricing," The Bell Journal of Economics, , 197-206.

MacKie-Mason, J. K. (1987) "Notes on Welfare Effects of Optional Peak-Load Pricing," University of Michigan Working Paper.

Mirman, L. J. and D. Sibley (1980) "Optimal Nonlinear Prices for Multiproduct Monopolies," Bell Journal of Economics, 11, 659-670.

Steiner, P. (1957) "Peak Loads and Efficient Pricing," Quarterly Journal Of Economics, 71, 585-610.

Turvey, R. (1968) "Peak Load Pricing," Journal of Political Economy, 76, 101-113.

Wenders, J. (1976) "Peak load pricing in the Electric Utility Industry," The Rand Journal of Economics, , 232-241.

Williamson, O. (1966) "Peak-Load Pricing and Optimal Capacity Under Indivisibility Constraints," American Economic Review, 56, 810-82i.

Willig, R. D. (1979) "Pareto-Superior Nonlinear Outlay Schedules." Bell Journal of Economics, 9, 56-69. 


\section{Recent Crest Working Papers}

88-1: Carol A. Jones, Suzanne Scotchmer, "The Social Cost of Uniform Regulatory Standards in a Hierarchical Government" December, 1987.

88 2: 'Ted Bergstrom, Judy Roberts, Dan Rubinfeld, Perry Shapiro, "A Test for Efficiency in the Supply of Public Education" December 12, 1987.

88 3: Mark Bagnoli, J. Bradley Barbeau, "Competition and Product Line Choice" February, 1988.

88-4: Severin Borenstein, Paul N. Courant, "How to Carve a Medical Degree: IIuman Capital Assets in Divorce Settlements" December, 1987.

88-5: Mark Bagnoli, Stephen W. Salant, Joseph E. Swierzbinski, "Pacman Refutes the Coase Conjecture: Durable-Goods Monopoly with Discrete Demand" January, 1988.

88 6: Jonathan Cave, Stephen W. Salant, “A Median Choice Theorem” December 29, 1987.

88*7: Mark Bagnoli, Naveen Khanna, "Why Are Buyers Represented by Seller's Agents When Buying a IIouse?" December, 1987.

88 8: Mark Bagnoli, Roger Gordon, Barton L. Lipman, "Takeover Bids, Defensive Stock Repurchases, ancl the Efficient Allocation of Corporate Control" October, 1987.

88- 9: Mark Bagnoli, Barton L. Lipman, "Private Provision of Public Goods can be Efficient" November, 1987.

88 10: Michelle J. White, "Urban Commuting Journeys are Not "Wasteful"” February, 1988.

88 11: A very Katz, "A Note on Optimal Contract Damages When Litigation is Costly" February, 1988.

88 12: 'l'ed Bergstrom, Jeffrey K. MacKic-Mason, "Notes on Peak Load Pricing" l'ebruary, 1988.

88. 13: Jerry A. Ilausman, Jeffrey K. MacKie-Mason, "Price Discrimination and Patent Policy" February, 1988 .

89 01: Mark Bagnoli, Scverin Borenstein, "Carrot and Yardstick Regulation: Enhancing Market Performance with Output Prizes" October, 1988.

89. 02: Ted Bergstrom, Jeffrey K. MacKie-Mason, "Some Simple Analytics of Peak-Load Pricing" October, 1988.

89 03: Ken Binmore, "Social Contract I: Harsanyi and Rawls" June, 1988.

89 01: Ken Binmore, "Social Contract II: Gauthicr and Nash" June, 1988.

89 05: Ken Binmore, "Social Contract III: Evolution and Utilitarianism" June, 1988.

89 06: Ken Binmore, Adam Brandenburger, "Common Knowledge and Game Theory" July, 1988.

89 07: Jeffrey A. Miron, "A Cross Country Comparison of Seasonal Cycles and Business Cycles" November, 1988 .

89-08: Jeffrey A. Miron, "The Founding of the Fed and the Destabilization of the Post-1914 Economy" August, 1988.

89 09: Gerard Gaudet, Stephen W. Salant, "The Profitability of Exogenous Output Contractions: A Comparativi:Static Analysis with Application to Strikes, Mergers and Export Subsidies" July, 1988.

89-10: Gerard Gaudet, Stephen W. Salant, "Uniqueness of Cournot Equilibrium: New Results from Old Methods" August, 1988.

89 11: Ilal R. Varian, "Goodness-of-Fit in Demand Analysis" September, 1988.

89 12: Michelle J. White, "Legal Complexity" October, 1988.

89 13: Michelle J. White, "An Empirical Test of the Efficiency of Liability Rules in Accident Law" November, 1988.

89-11: Carl P. Simon, "Some Fine--Tuning for Dominant Diagonal Matrices" July, 1988. 



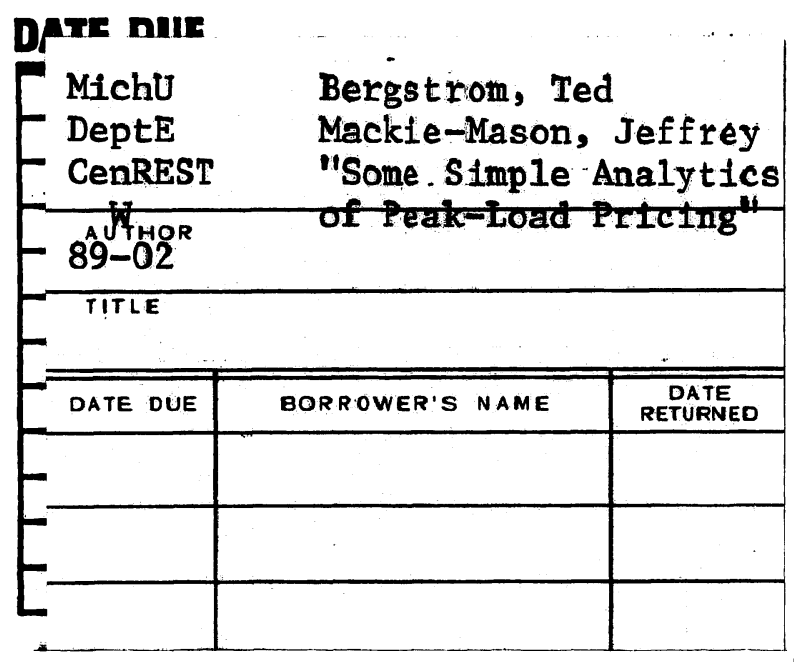


